\title{
Incidence of Hepatitis D Virus Infection in Japanese Patients with Hepatocellular Carcinoma
}

\section{- Immunohistochemical Investigation of the Delta Antigen--}

\author{
MASAYOSHI KAGE, KENICHIRO KOSAI, KAZUHIDE SHIMAMATSU, \\ OSAMU NAKASHIMA, MAKOTO HARAMAKI, \\ MASAFUMI YASUNAGA, HAYATO IHA, \\ MASAMICHI KOJIRO AND SUGANTHA GOVINDARAJAN* \\ Department of Pathology, Kurume University School of Medicine, Kurume, 830 Japan \\ and *Department of Pathology, Rancho Los Amigos Hospital, University of \\ Southern California, Downey, California, 90242 U.S.A.
}

Received for publication October 26, 1992

\begin{abstract}
Summary: In order to clarify the incidence of the delta agent among hepatocellular carcinomas (HCCs) in Kurume, where the hepatitis B infection and its related HCC are most prevalent in Japan, liver tissues from sero hepatitis B surface antigen-positive autopsy cases, with or without HCC, were immunohistochemically investigated for detection of the delta antigen. Only one patient (1.7\%) among 58 patients with $\mathrm{HCC}$ was found to have deltaantigen in the nuclei in the hepatocytes, which were diffusely distributed throughout the non-cancerous liver. None of 26 patients with liver cirrhosis showed delta-antigen in the liver tissue. The incidence is so low that the delta agent is unlikely to have a role in the development 'of HCC in our areas.
\end{abstract}

Key words: hepatitis B - delta virus - hepatocellular carcinoma - immunohistochemistry - liver cirrhosis

\section{Introduction}

The delta agent, first described in Italy, has been found around the world, although with varying incidence in different geographical regions (Rizzetto et al. 1980). Southeast Asia is an area where hepatitis B carriers are frequently found. However, there are only a few reports of delta infection there, suggesting low incidence of delta infection (Rizzetto et al. 1980; Omata et al. 1985; Govindarajan et al. 1989; Yano et al. 1989). It has also been reported that the incidence of delta infection in Japan is very low (less than
$2 \%$ ) in hepatitis B surface antigen (HBsAg) positive patients with acute or chronic liver disease (Rizzetto et al. 1980; Omata et al. 1985; Yano et al. 1989). Thus, less than thirty patients with hepatitis delta virus (HDV) infection have been reported. Therefore, probably due to such a low incidence of delta infection in Japan, little attention has been paid to HDV infection among patients with hepatocellular carcinoma (HCC) in Japan. Despite this, about one hundred thousands people annually die of HCC, of whom $20-40 \%$ are estimated to be hepatitis B carrier. To our knowledge, only

Address correspondence: Masayoshi Kage, M.D., The First Department of Pathology, Kurume University School of Medicine, 67 Asahi-machi, Kurume, 830 Japan. 
two Japanese patients with $\mathrm{HCC}$ related to delta agent were reported so far (Yano et al. 1989), and no histological investigations of the delta antigen in a large number of HCC have been carried out in Japan. The aim of this study is to clarify the prevalence of the delta agent among Japanese patients with HCC.

\section{Materials and Methods}

\section{Patients}

Liver specimens from autopsied patients with positive HBsAg in serum were collected from the autopsy file of the Department of Pathology of Kurume University School of Medicine for the past 14 years (1976-1989). HBsAg was measured by the reversed passive hemagglutination (PHA) method (Abbott, Lab, North Chicago, IL). The delta-antigen in the liver tissue was studied in $84 \mathrm{HBs} A g$ seropositive patients with chronic liver diseases. They consisted of 26 patients with liver cirrhosis and 78 patients with liver cirrhosis associated with HCC. There were 82 males and 22 females with a mean age of 59.7 years. None was a known intravenous drug user or homosexual.

\section{Immunohistochemistry}

One or two liver tissue specimens contained a tumor taken from a formalinfixed autopsy liver. The liver sections were embedded in paraffin, cut into thin sections, and stained for delta-antigen using indirect immunoperoxidase and peroxidase-antiperoxidase techniques (Dako Co., Santa Barbara, CA) as previously described in detail (Govindarajan et al. 1984a). Specificity of staining was comfirmed by blocking with unlabeled human anti-delta serum.

\section{Results}

The delta-antigen was found in the liver in one of the $104(0.7 \%)$ patients with HCC. None of 26 patients with liver cirrhosis manifested the delta-antigen in the liver. The delta-antigen positive patient was a 41 year-old male who developed ascites and hypoproteinemia in November, 1977 and suffered from repeated hematemesis from esophageal varices. The patient died of massive gastrointestinal hemorrhage in May, 1978. Serum HBsAg was positive. The patient had no history of previous jaundice, intravenous drug use, or blood transfusion. This patient made a short trip to Hawaii in May, 1975 for sightseeing. The autopsy revealed that the liver, weighing $1400 \mathrm{~g}$, showed advanced cirrhosis of mixed nodular type complicated with HCC. Deltaantigen was positively stained in the nuclei of the hepatocytes, which were diffusely scattered throughout the non-cancerous liver. No delta-antigen was observed in HCC. The liver in non-cancerous regions also showed unevenly distributed hepatitis B core antigen-positive hepatocytes.

\section{Discussion}

This retrospective investigation shows low frequency of intrahepatic delta-antigen in livers with or without HCC. This indicates that the HDV does not have significant role in the development of $\mathrm{HCC}$ in most cases in the Kurume area. Our finding of low prevalence of intrahepatic delta-antigen in $\mathrm{HCC}$ is in agreement with other studies using immunohistochemistry (Craxi et al. 1983; Hadziyannis et al. 1983; Govindarajan et al. 1984b). In the United State, intrahepatic delta-antigen was found in only one of 39 specimens bearing HCC (Govindarajan et al. 1984b), and in Greece it was present in 3 of 24 carriers with HCC (Hadziyannis et al. 1983). In a series from Italy, the antigen was absent 
in 18 cases of HCC (Craxi et al. 1983). Thus, there appears to be a low correlation between the presence of intrahepatic delta-antigen and HCC in those areas where high prevalence of HDV infection was reported. However, more recent studies in a larger number of cases have shown a comparable prevalence of anti-HDV in patients with HCC and chronic liver disease (Ponzetto et al. 1988; Oliveri et al. 1991). Further investigations are needed to clarify the relationship between the importance of delta infection and development of HCC.

It is of interest that the delta-antigen positive patient in our series died at age of 41 which was about 20 years younger than most patients with HCC in our autopsy cases, with an average age of 61.7 years. It is suggested that patients may die earlier due to aggravation of the cirrhotic liver disease process by the delta agent superinfection before HCC develops (Pagliaro et al. 1983). Recently, Oliveri (1991) reported that patients with HCC and HDV infection were significantly younger than those with HCC but lacking the HDV infection. They speculated that the rapid establishment of liver cirrhosis caused by HDV superinfection may function as an indirect promoting factor of the early development of HCC.

Our investigation also revealed that the HDV infection did exist among native Japanese living in the Kurume area in 1977. Although the mode of delta virus infection in this patient was unclear, it is possible that he was infected with delta agent when he went abroad. Recently, the number of Japanese going abroad has been rapidly increasing to reach 11 million in 1991, about two times the number in 1986. Subsequently, at least 100,000 Japanese hepatitis B carriers estimated from a 1-2\% carrier rate in a Japanese population travel to foreign countries every year. Visitors from foreign countries have also been increasing. Thus, a risk of introduction of the HDV into the Japanese population is increasing, and because of its strong infectivity, may lead to epidemic outbreak of HDV infection. An outbreak of the HDV infection in an isolated setting with a high HBV carrier rate was reported among Yucpa Indians in western Venezuela (Hadler et al. 1984) and in another part of South America (Buitrago et al. 1986). This study indicated that any population having a high prevalence of hepatitis B carriers may be at risk of an outbreak of severe hepatitis through non-drug users.

\section{References}

Buitrago, B., Popper, H. and Hadler, S.C. (1986). Specific histologic features of Santa Marta hepatitis: a severe form of hepatitis $\delta$-virus infection in Northern South America. Hepatology 6, 1285-1291.

Craxi, A., Raimondo, G. and Giannuoli, G. (1983). Delta agent and hepatocellular carcinoma. In Delta Infection and Viral Hepatitis. Progress in Clinical and Biological Research, ed. Verme, G., Bonino, F. and Rizzeto, M., pp. 231-234. New York: Alan R Liss.

Govindarajan, S., Hevia, F.J. and Peters, R. L. (1984a). Immunohistochemical localization of the delta antigen associated with hepatitis B virus in liver biopsy sections embedded in Araldite. Histopathology 8, 63-67.

Govindarajan, S., Hevia, F. J. and Peters, R.L. (1984b). Prevalence of delta antigen/antibody in $\mathrm{B}$ viral induced hepatocellurar carcinoma. Cancer 53, 1692-1694.

Govindarajan, S., Lee, S.D., Tong, M. J., Tsai, Y. T. and Lo, K. J. (1984). Prevalence of delta agent among Chinese in Taiwan and Los Angeles. J. Medical Biology 14, 33-37.

Hadler, S. C., Monzon, M. and Ponzetto, A. (1984). Delta virus infection and severe hepatitis: an epidemic in the Yucpa Indian Venezuela. Ann. Int. Med. 100, 339-343.

Hadziy annis, S. J. (1983). Delta antigen positive chronic liver disease in Greece: clinical aspects and natural course. In Delta Infection and Viral Hepatitis. Progress in Clinical and Biological Research, ed. Verme, G., Bonino, F. 
and Rizzeto, M., pp. 209-217. New York: Alan $\mathrm{R}$ Liss.

Oliveri, F., Brunetto, M.R., Baldi, M., Piantino, P., Ponzetto, A. et al. (1991). Hepatitis delta virus (HDV) infection and hepatocellular carcinoma (HCC). Prog. Clin. Biol. Res. 364, 217-222.

Omata, M., Ito, Y. and Imazeki, F. (1985). Infection with delta agent in Japan. Hepatogastroenterology 32, 220-223.

Pagliaro, L., Simonetti, R.G., Craxi, A., Spano, C. and Filippazzi, M.G. (1983). Alcohol and HBV infection as risk factors for hepatocellular carcinoma in Italy: a multicentric, controlled study. Hepato-gastroenterol, 30, 48-50.

Ponzetto, A., Hele, C., Forzani, B., Avanzini, L. and Rizzetto, M. (1988). Hepatitis Delta virus and hepatocellular carcinoma. Gastroenterology 94, 583-589.

Rizzetto, M., Purcell, R.H. and Gerin, J.L. (1980). Epidemiology of HBV-associated delta agent: geographical distribution of anti-delta and liver disease in drug-addicts. Lancet 1, 1215-1219.

Yano, M. and Imanami, E. (1989). Hepatitis D. Igaku no Ayumi 151, 766-770. (in Japanese) 